\title{
Sensitive monitoring of RNA transcription levels using a graphene oxide fluorescence switch
}

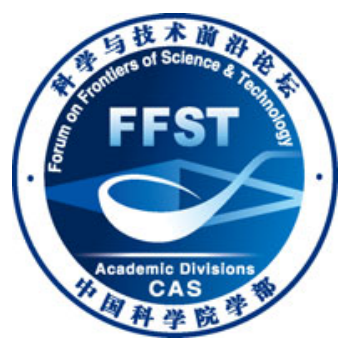

\author{
ZHOU XiaoMing, LIAO YuHui \& XING Da* \\ Key Laboratory of Laser Life Science \& Institute of Laser Life Science (Ministry of Education), College of Biophotonics, South China Normal \\ University, Guangzhou 510631, China
}

Received July 18, 2012; accepted October 26, 2012; published online December 26, 2012

\begin{abstract}
Catalytic transfer of genetic information from DNA to RNA is very important in life activities. The unconventional RNA transcription level may be related to the source of genetic diseases. At present, conventional methods for detection of RNA transcripts usually involve cumbersome preparative steps, or require sophisticated laboratory equipments. In this study, we presented a rapid, sensitive nano-detection platform for monitoring of RNA transcript levels. T7 RNA polymerase transcription reaction is employed as the example to test the feasibility of this method. In this design, in vitro synthesized RNA products can be hybridized to the FAM labeled single strand DNA (ssDNA) probes which can be adsorbed onto the graphene oxide (GO) surface. Using GO as the fluorescence switch, excellent capacity of the signal-on fluorescence platform for detection of RNA transcripts level is demonstrated. Transcription levels sensing with this nano-platform achieved a sensitivity of $5 \mathrm{pmol} / \mathrm{L}$ transcription template. It is anticipated that current developed RNA transcript nano-detection mode has the potential to be an alternative to the conventional RNA transcript detection methods.
\end{abstract}

nanobiotechnology, graphene oxide, fluorescence resonance energy transfer, RNA transcription level

Citation: Zhou X M, Liao Y H, Xing D. Sensitive monitoring of RNA transcription levels using a graphene oxide fluorescence switch. Chin Sci Bull, 2013, 58: 2634-2639, doi: 10.1007/s11434-012-5584-2

Catalytic transfer of genetic information from DNA to RNA is one of the most grounded life processes [1]. In the transcription reaction, DNA is used as the template, and the genetic information is passed to the RNA genome. RNA transcription is the genetic basis of many important in vivo life activities. This is because abnormal expression of genes usually caused protein dysfunction. At present, it is believed that the transcription levels of gene expression profiles from the mRNA levels can reflect the activities of abnormal cells in early disease development [2-7].

At present, methods for detection of RNA transcripts level include Northern blot, dot blot, reverse transcription polymerase chain reaction (RT-PCR), microarray, in situ hybridization method, and so on. Northern blot and dot blot involve cumbersome experiment steps during the operation. Moreover, RNA is susceptible to the RNA enzyme degrada-

*Corresponding author (email: xingda@scnu.edu.cn) tion, thus requires strict requirements for laboratory equipment and the environment, which may lead to the poor experimental reproducibility. Reverse transcription-PCR method is relatively simple and sensitive. However, electrophoresis detection takes a long time and need to use toxic reagents. Microarray and in situ hybridization method requires sophisticated equipment, and is not conducive to the adoption and using of the technology. Another potential application of the transcription levels detection is that isothermal transcription of the nucleic acid can be used as a signal amplification platform [8]. By the conjugation of nucleic acids and antibody, the linked nucleic acid can be transcribed into thousands of single strand RNA molecules. Through the detection of transcription products, more than one thousand times signal amplification can be achieved in protein detection [8,9]. Recently, some high-sensitivity biological analysis tools were developed based on transcription mediated amplification [8-15]. 
For these reasons, the development of new detection methods for the transcriptional level will play a vital role in the interpretation of specific functional gene regulation mechanisms, the elucidation of molecular basis of disease caused by gene expression abnormalities, and the drug target discovery and high throughput drug screening. Furthermore, novel transcriptional levels monitoring method can also provide high sensitivity analytical tool for bio-sensing at the single molecule level [16].

With the development of nanotechnology, nanomaterial has become an important tool in biotechnology research. In recent years, due to its excellent physical and chemical properties, graphene oxide $(\mathrm{GO})$ has gained tremendous interests in the biosensor fields for biosensors building. GO is constituted by single-layer sheet structure carbon atoms [17-22], with carboxyl and phenol hydroxyl group on its surface. GO has a net negative charge, good dispersion, and excellent stability [20]. It is also well known that singlestranded nucleic acid is soft and loose linear structure, thus adsorption of it onto the GO is stable, due to $\pi-\pi$ stacking effect between the nucleic acid base and GO [23,24]. However, the formation of the double helix nucleic acid structure is rigid, therefore greatly reduces its adsorption capacity by the GO $[23,24]$. On the other hand, one interesting property of GO is that it can be used as a broad-spectrum fluorescence quenching agent [23-29]. Therefore, fluorophores labeled in single-stranded nucleic acid probe can be effectively quenched by GO through fluorescence resonance energy transfer (FRET) mechanism. This distinct function derived from its heterogeneous chemical atomic structure and electronic properties, and the $s p 2$ hybrid crystal domain of $\mathrm{GO}$ is believed to play leading role in fluorescence quenching [17]. This quenching effect can be used as a fluorescence switch, thereby enhancing the signal to noise ratio of biological sensing. Based on this principle, a series of graphene-based bimolecular sensing technologies have been developed in recent years [23-29].

The utilization of GO allows the attachment of the functional nucleic acids probe onto its surface, thus providing the opportunity to construct a new strategy to detect RNA transcription levels. In this design, fluorophore labeled in the DNA probe acting as the energy donor and by functioning GO as the energy acceptor. Here, we chose T7 RNA polymerase transcription reaction to test the feasibility of this method. The results demonstrated that the GO fluorescence switch can be an excellent and versatile transcription monitoring platform. It is anticipated that currently developed RNA transcript nano-detection modes have the potential to be an alternative to the conventional RNA transcript detection methods.

\section{Materials and methods}

\subsection{Reagents and apparatus}

(1) Reagents. Graphene oxide $(1 \mathrm{mg} / \mathrm{mL})$ was purchased from Xianfeng Nanotechnologies Co., Ltd (Nanjing, China). T7 RNA polymerase and ribonucleotide triphosphate (rNTPs) mix were the products of New England Biolabs (NEB). SYBR I and SYBR II were purchased from Invitrogen. All oligonucleotides and probes used in our research are synthesized and purified by HPLC at Shanghai Sangon Biological Engineering and Technology \& Services Co., Ltd. The regents related to electrophoresis were purchased from Bio-Rad (Richmond, CA, USA). SSC buffer and RNasefree water were purchased from Shanghai Sangon Biotechnology Co. Ltd.

(2) Apparatus. Perkin-Elmer LS55 luminescence spectrometer (USA); fluorescence microplate reader (Infinite M200, TECAN); slab electrophoresis system and imaging system (Bio-Rad).

\subsection{T7 RNA transcription system}

The transcription system contained DNA template, rNTPs $\operatorname{mix}(2 \mathrm{mmol} / \mathrm{L}), \mathrm{T} 7 \mathrm{RNA}$ polymerase $(2.5 \mathrm{U} / \mu \mathrm{L}), 1 \times$ corresponding buffer, and ribonuclease inhibitor $(1 \mathrm{U} / \mu \mathrm{L})$. The DNA template was two single strands obtained by gradient cooling (denatured at $95^{\circ} \mathrm{C}$ for $5 \mathrm{~min}$, then cooling to $25^{\circ} \mathrm{C}, 2^{\circ} \mathrm{C}$ per min). Their sequences were 5'-CGCGAAATTAATACGACTCACTATAGGGAGA-3' (coding strand) and 5'-AACTTTCAACATCAGTCTGATAAGCTATCTCCCTATAGTGAGTCGTATTAATTTCGCG-3' (template strand). The transcription mixture was incubated for $2 \mathrm{~h}$ at $37^{\circ} \mathrm{C}$.

\subsection{Polyacrylamide gel electrophoresis}

Transcription products were analyzed on a Bio-Rad (BioRad Laboratories, USA) slab electrophoresis system. The $10 \mu \mathrm{L}$ samples were loaded onto a $10 \%$ native polyacrylamide gel (29:1, acryl:bisacryl) in $0.5 \times$ Tris-borate-EDTA (TBE). Gels were run at room temperature for $1 \mathrm{~h}$ at $120 \mathrm{~V}$. The gel was confirmed by SYBR I and SYBR II staining and photographed by Bio-Rad digital imaging system.

\subsection{GO nano-detection platform}

The steps were as follows: the single-stranded RNA product from transcription was dissolved in $1 \times \mathrm{SSC}$ buffer and FAM probe was also added to make a final concentration of $50 \mathrm{nmol}$. The resulting mixture was incubated for $30 \mathrm{~min}$ at $37^{\circ} \mathrm{C}$, and then FAM probe would hybridize with RNA product. Afterwards, GO was added to make a final concentration of $8 \mu \mathrm{g} / \mathrm{mL}$. After incubating for $10 \mathrm{~min}$, the fluorescence was measured with luminescence spectrometer. The sequence of FAM probe was 5'-TTTCAACATCAGTCTGATAAGCTATCTCCC-3', and the $3^{\prime}$ terminal was labeled with FAM fluorescent (excitation at $488 \mathrm{~nm}$ and emission at $520 \mathrm{~nm}$ ). 


\section{Results and discussions}

\subsection{Design of the GO fluorescence switch strategy}

This study aims to build a fast, sensitive detection tool for RNA transcript levels with the GO fluorescent switch. The platform is based on nucleic acid hybridization and FRET principle. We used T7 RNA polymerase transcript reaction as the example to test the feasibility. T7 RNA polymerase, encoded by $\mathrm{T} 7$ bacteriophage [30,31], is highly specific to the promoter sequence (TAATACGACTCACTATAGGGAGA). The enzyme is widely used in the isothermal amplification of nucleic acids. In T7 RNA polymerase transcription, double-stranded template DNA is necessary for the promotor region, but the transcription region only needs the single strand as the template. Therefore, in this study singlestranded template strand is employed in the transcribed region. The experimental principle is shown in Figure 1. First, T7 RNA polymerase binds to the promotor region of the template. Adding of the rNTPs leads to the continuous transcription. A FAM labeled DNA probe was designed to hybridize to the corresponding single-stranded RNA transcripts. GO has been demonstrated interacting strongly with nucleotides through $\pi$-stacking interaction. However, adsorption of double stranded DNA (dsDNA) onto the GO surface is weak, due to the shielding of nucleobases within the negatively charged phosphate backbone of dsDNA. Furthermore, GO can quench the fluorescence of the nearby dyes. By monitoring the increase in fluorescence intensity, the dynamics of transcription reaction can be detected with very high sensitivity.
In order to verify the validity of the $\mathrm{T} 7$ transcription reaction, $50 \mathrm{nmol} / \mathrm{L}$ DNA template is transcribed by T7 RNA polymerase. The transcripts were detected using $12 \%$ polyacrylamide gel electrophoresis. Figure 2 shows that the RNA transcripts can be stably produced from three paralleled transcription experiments.

\subsection{Parameters for graphene fluorescence switch detection of transcripts}

In building process of this nano-detection platform, it is found that the RNA products of transcription system can be adsorbed onto GO surface at high GO concentration, leading to desensitization of the nano-detection platform. However, when GO concentration is too low, single-stranded FAM probe cannot be completed adsorbed, resulting in a strong background fluorescence signal, thus to lower the signal to noise ratio. To get this optimized parameter, experiments were executed to evaluate the effect of GO concentration on the fluorescence quenching ability. FAM labeled DNA probe concentration was fixed for $50 \mathrm{nmol} / \mathrm{L}$ in all experimental groups. GO concentrations are 2, 4, 6, 8, 10 $\mu \mathrm{g} / \mathrm{mL}$, respectively. As shown in Figure 3, with the increase of the GO concentration, fluorescence quenching efficiency got increased, indicating that more FAM labeled DNA probe was adsorbed. When the GO concentration was increased to $8 \mu \mathrm{g} / \mathrm{mL}$, fluorescence quenching efficiency achieved a maximal value. Further increasing that the GO concentration quenching efficiency was not significantly improved. Therefore, it seems $8 \mu \mathrm{g} / \mathrm{mL}$ GO is the optimum choice for the subsequent nano-detection platform.
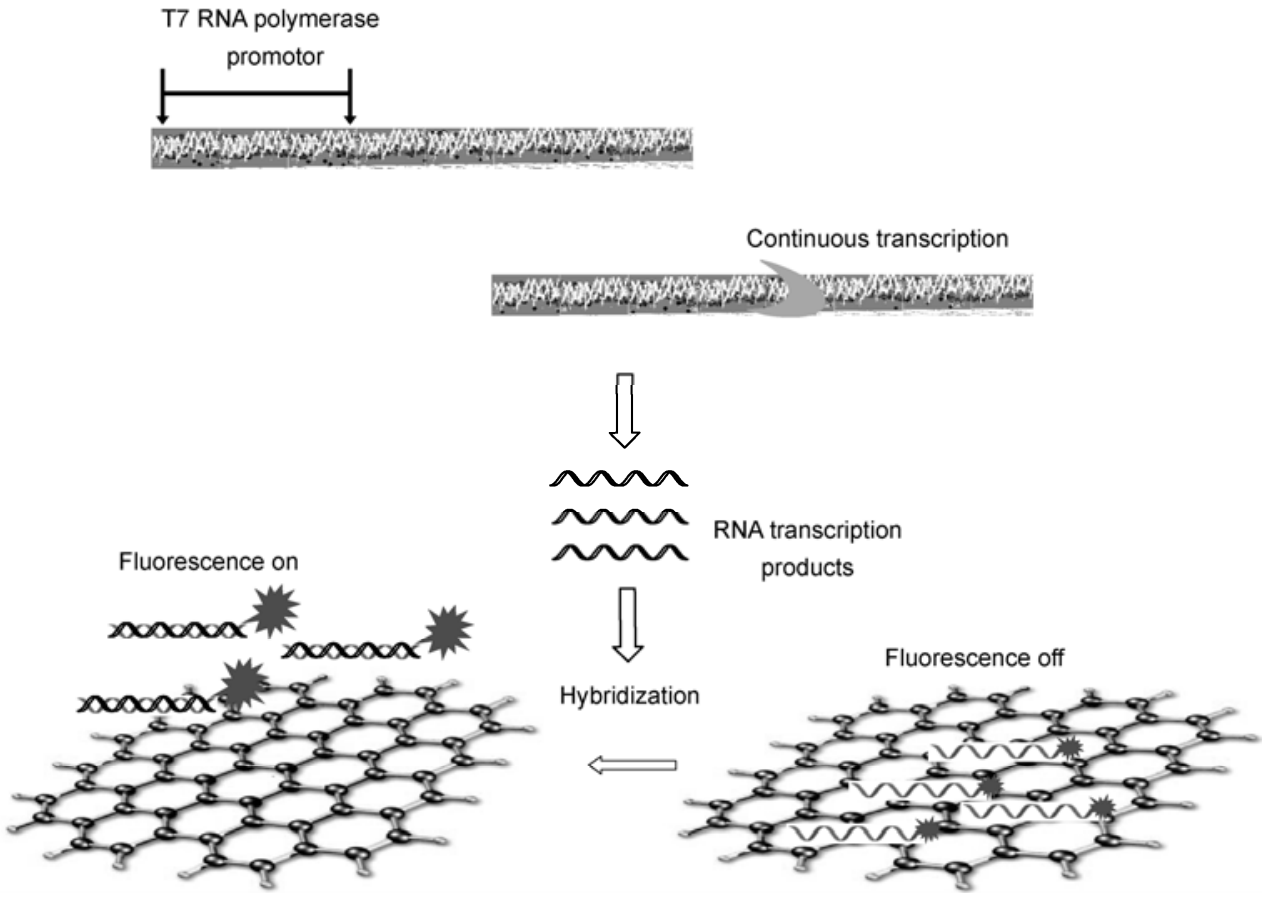

Figure 1 The principle of monitoring of RNA transcription levels using a GO fluorescence switch. 


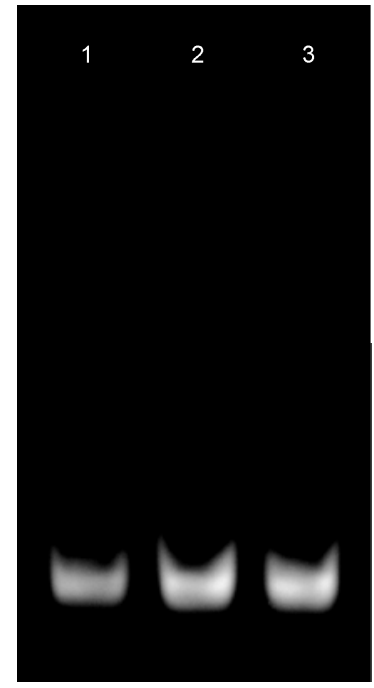

Figure 2 Electrophoresis identification of the T7 transcription reaction. The transcription products are separated by $12 \%$ polyacrylamide gel electrophoresis. DNA is screened with a SYBR GREEN I/II MIX staining method. Lanes 1 to 3 represent the three repeated experiments.

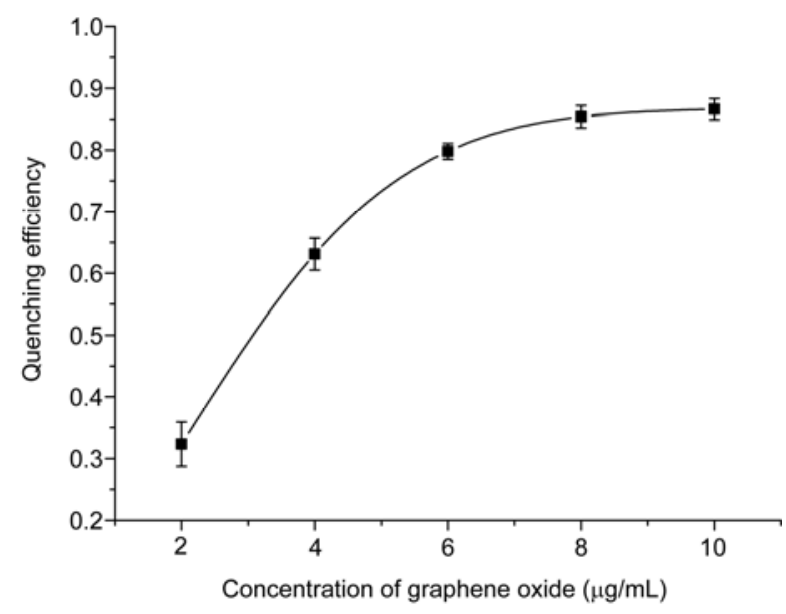

Figure 3 Evaluation of the effect of GO concentration on the fluorescence quenching ability. Optimal GO concentration is $8 \mu \mathrm{g} / \mathrm{mL}$.

On the other hand, time dynamics of fluorescent probes adsorption is also an important parameter. It is because singlechain fluorescent probes could not be effectively adsorbed onto GO if too short adsorption time is employed. However, random adsorption of double-stranded hybrid products may occur when long incubation time is used, which reduces the signal to noise ratio. In order to improve the detection efficiency of the nano-detection platform, the GO adsorption time optimization experiments were executed. FAM labeled DNA probe concentration is fixed at $50 \mathrm{nmol} / \mathrm{L}$, GO concentration is $8 \mu \mathrm{g} / \mathrm{mL}$, and adsorption time variable detection of the fluorescence intensity is observed at different time point. The experimental results are shown in Figure 4. It is found that with the increase of adsorption time, the fluorescence quenching efficiency gradually increased. When the adsorption time was $8 \mathrm{~min}$, the fluorescence quenching efficiency keeps stabilized. Therefore, 8 min was chosen as optimal adsorption time in subsequent experiments.

\subsection{Sensitivity results and data analysis}

In order to verify the sensitivity of GO nano-detection platform for RNA transcripts, transcription templates sensitivity experiments were designed. DNA transcription template was varied from the concentration of $0.5,5,50,500 \mathrm{pmol} / \mathrm{L}$, $5 \mathrm{nmol} / \mathrm{L}$, to $50 \mathrm{nmol} / \mathrm{L}$, respectively. Transcription time was $2 \mathrm{~h}$. Transcripts derived from different template concentrations were monitored by GO nano-detection platform. Results are shown in Figure 5. It is found that fluorescence intensity gradually increased with the increasing concentration of DNA transcription template. Further increase of the concentration of the DNA transcription template to $5 \mathrm{nmol} / \mathrm{L}$ did not significantly enhance the fluorescence intensity, suggesting that $5 \mathrm{nmol} / \mathrm{L}$ DNA template has reached the saturation limit of the GO nano-platform testing. When the concentration of DNA transcription template is $0.5 \mathrm{pmol} / \mathrm{L}$, similar fluorescence intensities are observed between experimental group and control group. However, with $5 \mathrm{pmol} / \mathrm{L}$ transcription template in the transcription and detection system, the fluorescence intensity was significantly enhanced. Because more detailed transcription template concentration experiments are not executed, it is well demonstrated that current developed GO nano-detection platform achieved a sensitivity of $5 \mathrm{pmol} / \mathrm{L}$.

In order to verify the reliability of experimental data and the feasibility of this nanotechnology platform for quantitatively testing RNA transcription levels, a histogram is constructed and linear dependence of the fluorescence intensities of different DNA template concentrations was analyzed based on repeated experiments and statistics. The results are shown in Figure 5. A $R^{2}$ value of 0.967 was obtained from

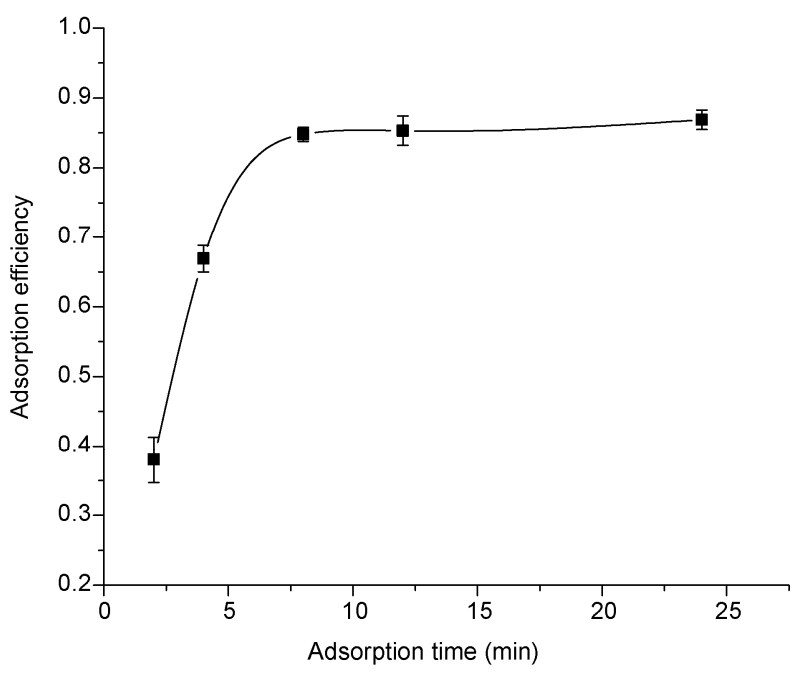

Figure 4 Time dynamics of fluorescent probes adsorption. GO concentration is $8 \mu \mathrm{g} / \mathrm{mL}$, FAM labeled DNA probe concentration was fixed for $50 \mathrm{nmol} / \mathrm{L}$. 


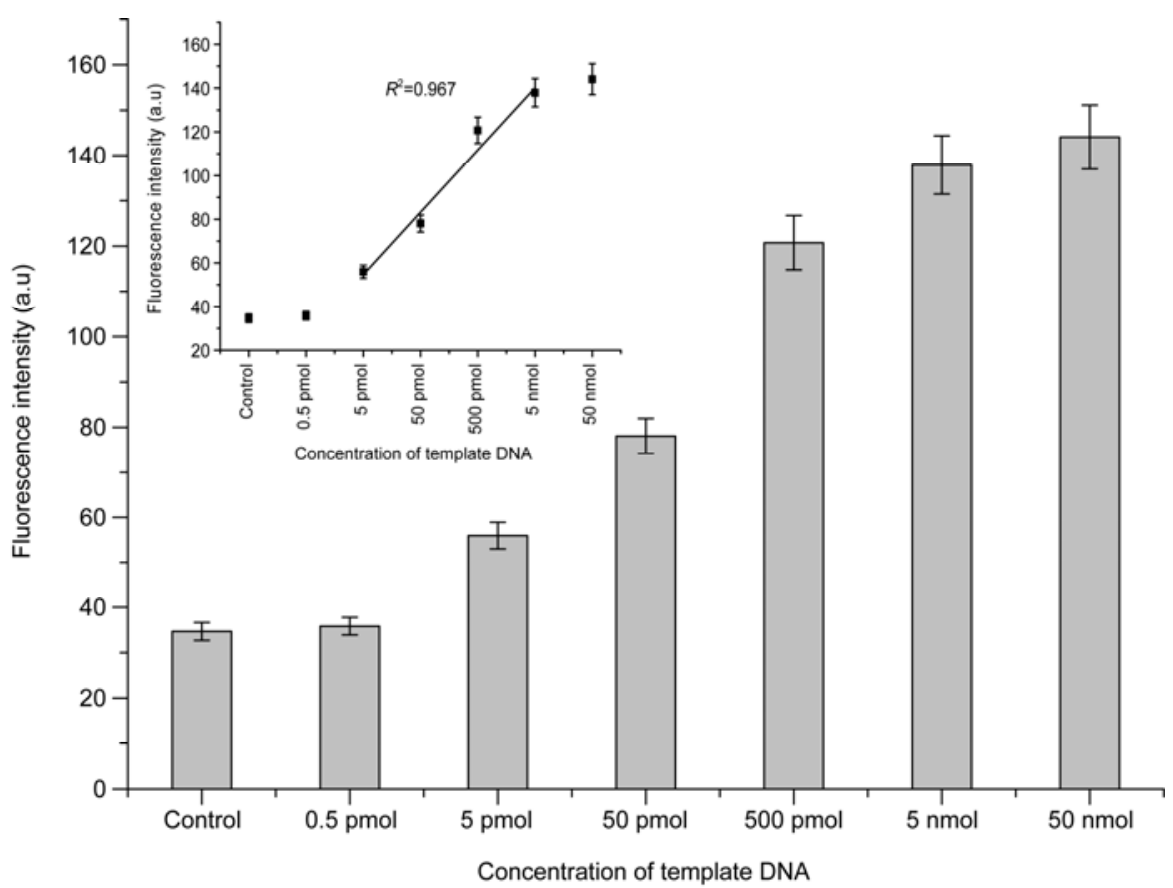

Figure 5 Fluorescence spectroscopy experiments for measuring transcripts levels derived from different concentrations of DNA transcription template. GO concentration is $8 \mu \mathrm{g} / \mathrm{mL}$, FAM labeled DNA probe concentration was fixed for $50 \mathrm{nmol} / \mathrm{L}$. Incubation time is $8 \mathrm{~min}$.

the concentration of $5 \mathrm{pmol} / \mathrm{L}$ to $5 \mathrm{nmol} / \mathrm{L}$. Based on this, it is well demonstrated the feasibility and reliability of the platform used for the detection of RNA transcript levels.

\section{Conclusions}

In summary, it is demonstrated that an approach can be potentially applied to the detection of RNA transcript levels. The proposed method is based on a GO fluorescence switch made of one dye labeled single stranded DNA obtained from conventional chemical synthesis and GO nanomaterial. Since GO is an excellent and broad spectrum fluorescencequencher, using $\mathrm{GO}$ as the fluorescence detection platform is an attractive option. The strategy to sensing RNA transcript levels with a dye-labeled DNA probe by hybridization also circumvents the processes of chemical modification and conjugation of GO.

Three remarkable advantages can be concluded when compared to some conventional assays. Firstly, the whole detection process is rapid. The 10-min incubation time is enough for effective hybridization of dye labeled DNA probes and RNA transcripts. Secondly, RNA transcripts from $5 \mathrm{pmol} / \mathrm{L}$ DNA template can be reliably detected, suggesting that current assay can be a robust tool for transcription-mediated amplification based bioassays. Thirdly, although single dye labeled probe is used for monitoring the RNA transcripts, the method has the potential to be a multicolor fluorescent RNA transcripts analysis. By employing of different dye labeled DNA probes, different RNA transcripts can be detected instantaneously in single tube reaction.
To the best of our knowledge, it is the first reported example of exploring nanomaterial for the transcripts levels monitoring. However, it is obvious that current strategy is not limited to the sensing of RNA transcription levels. One of the ideational examples is the sensing of the nucleic acid sequence based amplification (NASBA) reaction, which is an isothermal and sensitive nucleic acids detection technology having plenty of applications.

This work was supported by the National Basic Research Program of China (2010CB732602), the Key Program of NSFC-Guangdong Joint Funds of China (U0931005), the National Natural Science Foundation of China (81101121) and the Natural Science Foundation of Guangdong Province (S2011040005386)

1 Blake W J, Kaern M, Cantor C R, et al. Noise in eukaryotic gene expression. Nature, 2003, 422: 633-637

2 Bachl J, Carlson C, Schopfer V G, et al. Increased transcription levels induce higher mutation rates in a hypermutating cell line. J Immunol, 2001, 166: 5051-5057

3 Rahman I, MacNee W. Regulation of redox glutathione levels and gene transcription in lung inflammation: Therapeutic approaches. Free Radic Biol Med, 2000, 28: 1405-1420

4 Nagalakshmi U, Wang Z, Waern K, et al. The transcriptional landscape of the yeast genome defined by RNA sequencing. Science, 2008, 320: 1344-1349

5 Pugliese A, Zeller M, Jr A F, et al. The insulin gene is transcribed in the human thymus and transcription levels correlate with allelic variation at the INS VNTR-IDDM2 susceptibility locus for type 1 diabetes. Nat Genet, 1997, 15: 293-297

6 Terry C F, Loukaci V, Green F R. Cooperative influence of genetic polymorphisms on interleukin 6 transcriptional regulation. J Biol Chem, 2000, 275: 18138-18144

7 Jenssen T K, Lægreid A, Komorowski J, et al. A literature network of 
human genes for high-throughput analysis of gene expression. Nat Genet, 2001, 28: 21-28

8 Zhang H T, Kacharmina J E, Miyashiro K, et al. Protein quantification from complex protein mixtures using a proteomics methodology with single-cell resolution. Proc Natl Acad Sci USA, 2001, 98: 54975502

9 Tannous B A, Laios E, Christopoulos T K. T7 RNA polymerase as a self-replicating label for antigen quantification. Nucleic Acids Res, 2002, 30: e140

10 Burbulis I, Yamaguchi K, Gordon A, et al. Using protein-DNA chimeras to detect and count small numbers of molecules. Nat Meth, 2005, 2: 31-37

11 Zhang $\mathrm{H} \mathrm{T}$, Cheng $\mathrm{X}$, Richter $\mathrm{M}$, et al. A sensitive and highthroughput assay to detect low-abundance proteins in serum. Nat Med, 2006, 12: 473-477

12 Kattah M V, Coller J, Cheung R K, et al. HIT: A versatile proteomics platform for multianalyte phenotyping of cytokines, intracellular proteins and surface molecules. Nat Med, 2008, 14: 1284-1289

13 Freudenberg J A, Bembas K, Greene M I, et al. Non-invasive, ultrasensitive, high-throughput assays to quantify rare biomarkers in the blood. Methods, 2008, 46: 33-38

14 Sendroiu I E, Gifford L K, Lupták A, et al. Ultrasensitive DNA microarray biosensing via in situ RNA transcription-based amplification and nanoparticle enhanced SPR imaging. J Am Chem Soc, 2011, 133: 4271-4273

15 Geim A K, Novoselov K S. The rise of graphene. Nat Mater, 2007, 6: 183-191

16 João R, João C, Jesus M D I F, et al. Gold-nanobeacons for real-time monitoring of RNA synthesis. Biosen Bioelectron, 2012, 36: 161-167

17 Lü K, Zhao G X, Wang X K. A brief review of graphene-based mAterial synthesis and its application in environmental pollution management. Chin Sci Bull, 2012, 57: 1223-1234

18 Liu Z B, Zhang X L, Yan X Q, et al. Nonlinear optical properties of graphene-based materials. Chin Sci Bull, 2012, 57: 2971-2982

19 Xu M S, Gao Y, Yang X, et al. Unique synthesis of graphene-based materials for clean energy and biological sensing applications. Chin Sci Bull, 2012, 57: 3000-3009

20 Hea H Y, Klinowskia J, Forsterb M, et al. A new structural model for graphite oxide. Chem Phys Lett, 1998, 287: 53-56

21 Lerf A, He H, Forster M, et al. Structure of graphite oxide revisited. Phys Chem B, 1998, 102: 4477-4482

22 Lau P S, Coombes B K, Li Y F, et al. A general approach to the construction of structure-switching reporters from RNA aptamers. Angew Chem Int Ed, 2010, 49: 7938-7942

23 Lu C H, Yang H H, Zhu C L, et al. A graphene platform for sensing biomolecules. Angew Chem Int Ed, 2009, 48: 4785-4787

$24 \mathrm{Lu} \mathrm{C} \mathrm{H,} \mathrm{Li} \mathrm{J,} \mathrm{Lin} \mathrm{M} \mathrm{H,} \mathrm{et} \mathrm{al.} \mathrm{Amplified} \mathrm{aptamer-based} \mathrm{assay} \mathrm{through}$ catalytic recycling of the analyte. Angew Chem Int Ed, 2010, 49: 8454-8457

25 He S J, Song B, Li D, et al. A graphene nanoprobe for rapid, sensitive, and multicolor fluorescent DNA analysis. Adv Funct Mater, 2010, 20: 453-459

26 Li F, Huang Y, Yang Q, et al. A graphene-enhanced molecular beacon for homogeneous DNA detection. Nanoscale, 2010, 2: 10211026

27 Wang Y, Li Z H, Hu D H, et al. Aptamer/graphene oxide nanocomplex for in situ molecular probing in living cells. J Am Chem Soc, 2010, 132: 9274-9276

28 Dong H F, Gao W C, Yan F, et al. Fluorescence resonance energy transfer between quantum dots and graphene oxide for sensing biomolecules. Anal Chem, 2010, 82: 5511-5517

29 Wen Y Q, Xing F F, He S J, et al. A graphene-based fluorescent nanoprobe for silver (I) ions detection by using graphene oxide and a silver specific oligonucleotide. Chem Commun, 2010, 46: 2596-2598

30 Tabor S, Richardson C. A bacteriophage T7 RNA polymerase/promoter system for controlled exclusive expression of specific genes. Proc Natl Acad Sci USA, 1985, 82: 1074-1078

31 Kim J H, Larson R G. Single-molecule analysis of 1D diffusion and transcription elongation of T7 RNA polymerase along individual stretched DNA molecules. Nucleic Acids Res, 2007, 35: 3848-3858

Open Access This article is distributed under the terms of the Creative Commons Attribution License which permits any use, distribution, and reproduction in any medium, provided the original author(s) and source are credited. 